Chapter 2

\title{
Late-Onset Alzheimer's Disease: Risk Factors, Clinical Diagnosis and the Search for Biomarkers
}

\author{
Marisol Herrera-Rivero \\ Additional information is available at the end of the chapter \\ http://dx.doi.org/10.5772/53775
}

\section{Introduction}

Even with the progress that has been made in the past years on our understanding of Alzheimer's disease (AD) we don't seem to be closer to finding a cure than we were before. AD is a complex disorder wherein the pathophysiology is influenced by a great number of environmental and genetic factors, thus making it difficult to uncover the triggering events underlying disease's onset. In this chapter, we will first discuss some common conditions within the general population that have been associated with an increased risk to develop lateonset Alzheimer's disease (LOAD), such as hypertension, type 2 diabetes mellitus and high serum cholesterol and triglycerides levels, and the way they might be contributing to cognitive decline; depression and traumatic brain injuries, amongst others, will also be boarded.

Misdiagnosis is a frequent issue with important repercussions not only for the patient's condition but also for family members. For this reason, in the second part we will review the main basic aspects that should be involved in AD diagnosis, from laboratory tests to neuroimaging technologies, highlighting the importance of seeking a differential diagnosis of dementias in the elderly and the crucial role an accurate diagnosis may play in therapeutic outcomes as well as the implications for quality of life.

Early diagnosis of Alzheimer's may be the best tool we could find, as for now, to improve treatment outcomes by slowing the disease progression rate. This idea has led an important number of scientists around the globe to the search for biomarkers using a wide variety of approaches in the brain, cerebrospinal fluid (CSF) and blood. On this matter, we will briefly comment on our preliminary study using lymphocytes from cognitively healthy people and neuropsychological patients affected not only with LOAD but other types of neuropathologies as well to analyse in blood cells the expression of the main genes directly related to AD in brain cells. This study included 72 subjects in whom the expression of the microtubule-associated 
protein tau (MAPT), the amyloid precursor protein (APP), nicastrin (NCSTN), a component of the $\gamma$-secretase, and $\beta$-secretase (beta-site APP cleaving enzyme 1, BACE1) were analysed through 4 groups of cognitively healthy individuals ranging from 25 to 92 years of age and in conditions such as LOAD, vascular dementia (VaD) and Parkinson's disease (PD) in elderly individuals. We also aimed to discover the manner in which the expression of these genes might be affected by conditions associated with increased risk for LOAD such as hypertension and glucose, cholesterol and triglycerides serum levels.

\section{Risk factors for AD}

A wide variety of factors have been associated with an increased risk to develop LOAD; nevertheless, a number of these associations remain controversial. Age is the main risk factor to develop $\mathrm{AD}$ of the sporadic type and is only followed by apolipoprotein $\mathrm{E}$ (ApoE) genotype. However, other genetic and environmental conditions have also been proven to influence the risk of developing the disease and the rate of cognitive decline which affects disease progression. Amongst these are found cardiovascular risk factors, type 2 diabetes mellitus, sleep disorders, depression, education, smoking, alcohol, traumatic brain injury (TBI) and several single nucleotide polymorphisms (SNPs) in a growing list of candidate genes.

\subsection{Environmental}

a. Cardiovascular risk factors.

Within the risk factors for vascular disease hypertension and high cholesterol levels are most importantly associated with LOAD. Various longitudinal studies have shown an association between a diagnosis of hypertension in midlife and the development of AD in late life $[1,2]$. On this regard, hypertension may cause cerebrovascular disease which would contribute to accelerate $\mathrm{AD}$ processes, even when there are reports on a decrease in blood pressure during the disease course probably due to affection of the brain regions implicated in its regulation [3]. Cholesterol and its metabolism is associated with AD by several proposed mechanisms including regulation of beta-amyloid $(\mathrm{A} \beta)$ generation by an increase in $\beta$-secretase activity, APP membrane localization and cleavage variations by changes in cholesterol rich lipid rafts and ApoE-regulated interactions between cholesterol and $\mathrm{A} \beta$.

b. Type 2 diabetes mellitus.

Diabetes and even high glucose levels in the absence of a diabetes diagnosis are associated with an increased risk to develop AD involving various mechanisms, primarily being changes in the blood brain barrier (BBB) and the transport within cerebral small vessels [4].

c. Traumatic brain injury.

TBI has been associated with an increased risk to develop AD by longitudinal and retrospective studies, suggesting an effect between severity and repetitive episodes of TBI particularly by an increase in amyloid deposition [5, 6]. 


\section{d. Depression.}

It has been subject for debate whether depression might be an early manifestation of AD or a contributing factor for development of the disease. If one thing is sure is that depression contributes to cognitive decline and may by itself cause a condition known as "depressive pseudo-dementia" which can frequently evolve to a true dementia (mainly AD) when not treated, particularly in the elderly [7]. A history of major depressive disorder or susceptibility to depression during an individual's lifetime has therefore been associated with an increased risk to develop LOAD [8].

e. Sleep.

Chronic sleep deprivation and other sleep disorders have a negative effect on cognitive function primarily due to the role sleep plays in memory and learning processes and possibly in synaptic plasticity, although it is also believed there is an increase in $A \beta$ during the waking hours [9]. History of sleep disorders has thus been linked to an increase in the risk to develop LOAD; furthermore, sleep disorders occurring as part of the aging process and those related to coexisting medical conditions contribute to cognitive decline and behavioural problems in AD patients.

\section{f. Education.}

People with a lower level of education show an increased risk to develop LOAD. This might be due to the relative lack of constant stimulation of cognitive processes (such as learning and memory) compared to individuals with a higher education degree. In fact, cognitive stimulation therapies are widely used for helping the treatment of people with dementia [10].

g. Diet.

It is well known that diet affects every aspect of an individual's health. A number of medical conditions associated with an increased risk for developing AD have a strong nutritional background as cholesterol, glucose and vitamin $B_{12}$ levels, just to mention a few, importantly influence the risk for dementia [11], particularly in old age.

h. Alcohol and smoking.

It remains unclear whether alcohol and smoking increase the risk for $\mathrm{AD}$ as their association has been inconsistent. Although smoking is a strong risk factor for vascular disease, and the association of the latter with AD has been established, the possibility of a neuroprotective effect of nicotine by a smoking-induced increase in nicotinic receptors exists [3]. Similar is the case of alcohol where wine presents protective effects because of its antioxidant contents while associations between other types of alcohol and risk of AD remain controversial.

\subsection{Genetic}

Medical conditions and lifestyle in midlife, especially when a group of these factors coexist in an individual creating a synergistic effect, can contribute to cellular and molecular alterations ultimately leading to the hallmark pathological processes of AD later in life. Although 
environmental factors appear to play an important role in the development of LOAD, a number of them may depend on a genetic-influenced predisposition. Genetic variations in candidate genes have been widely investigated for associations with an increased risk of development, age of onset and progression of $\mathrm{AD}$ and, while results on several studies are controversial or inconclusive, it remains clear that common genetic variations do associate with LOAD whether in a general or population-specific manner. The AlzGene database holds information on 2973 polymorphisms in 695 genes reported in 1395 studies. We summarize in Table 1 some of the main findings on this field.

\begin{tabular}{|c|c|c|c|c|}
\hline Gene & Chromosome & SNP & Odds ratio & Studies \\
\hline APOE & 19 & $\mathrm{e} 2 / 3 / 4$ & 3.68 (4 vs. 3 ) & 37 \\
\hline BIN1 & 2 & rs744373 & 1.17 & 21 \\
\hline CLU & 8 & rs11136000 & 0.89 & 33 \\
\hline ABCA7 & 19 & rs3764650 & 1.23 & 10 \\
\hline CR1 & 1 & rs3818361 & 1.15 & 27 \\
\hline PICALM & 11 & rs3851179 & 0.88 & 27 \\
\hline MS4A6A & 11 & rs610932 & 0.90 & 11 \\
\hline CD33 & 19 & rs3865444 & 0.89 & 5 \\
\hline MS4A4E & 11 & rs670139 & 1.08 & 11 \\
\hline CD2AP & 6 & rs9349407 & 1.12 & 5 \\
\hline EPHA 1 & 7 & rs11767557 & 0.89 & 5 \\
\hline GSTO1 & 10 & rs4925 & 0.97 & 7 \\
\hline TOMM40 & 19 & rs8106922 & 0.66 & 7 \\
\hline SORL1 & 11 & rs2282649 & 1.10 & 23 \\
\hline PVRL2 & 19 & rs6859 & 1.50 & 8 \\
\hline NCSTN & 1 & rs2274185 & 0.90 & 5 \\
\hline BDNF & 11 & $270 \mathrm{C} / \mathrm{T}$ & 1.09 & 20 \\
\hline GAB2 & 11 & rs2373115 & 0.85 & 13 \\
\hline
\end{tabular}

Source: Meta-analysis for all studies from AlzGene-field synopsis of genetic association studies in AD (www.alzgene.org)

Table 1. Some widely studied genetic variations associated with an increased risk of AD.

At this point there is a need to highlight that prevention of LOAD may only be achieved by a healthy lifestyle implemented early in life, most importantly by those individuals with a genetic susceptibility. 


\section{Clinical diagnosis of LOAD}

To perform a clinical diagnosis of $\mathrm{AD}$ or other dementias may not be challenging when symptoms are from moderate to severe and well differentiated; however, it might be more difficult to achieve an accurate diagnosis in the early stages of the disease, especially in elderly individuals where initial manifestations of AD can be taken as an effect of aging. Early detection of cognitive decline in primary care thus becomes crucial for canalization of the patient to an adequate specialist and a subsequent early accurate diagnosis which allows a better management of disease progression. In the first stages of $A D$, memory processes are the most affected but since a number of other treatable medical conditions also present with deficiencies in memory performance, a differential diagnosis based on all available tools must always be pursued. Clinical diagnosis of LOAD should include laboratory and neuropsychological tests as well as structural and functional imaging of the brain which, together with the clinical history and interviews with those living closest to the patient, would provide a better idea of the patient's overall condition.

\subsection{Initial evaluation}

Clinical history and, importantly, interviews with close family members and friends provide valuable information on patient's medical history, lifestyle and disease onset and progression. Several issues may be considered though when evaluating an aged individual: clinical manifestations of psychiatric disorders differ from younger patients; life events, social and financial situations as well as physical status are also relevant aspects to be taken into account.

\subsection{Laboratory tests}

Running laboratory tests is important to identify secondary causes of dementia and medical conditions common in the aged population. It is advisable to perform a complete blood count, serum electrolytes, glucose, vitamin B12, BUN/creatinine ratio and thyroid and hepatic function panels. Electrocardiography (ECG), electroencephalography (EEG) and thorax $x$-rays can also be included. Increase in total tau protein (t-tau) and decrease in $A \beta_{42}$ in CSF are found in AD.

\subsection{Neuropsychological evaluation}

Neuropsychological evaluation is fundamental for dementia diagnosis providing evidence of cognitive dysfunction and specific patterns helping to uncover the cause. AD diagnosis is based on NINCDS-ADRDA (National Institute of Neurological and Communicative Disorders and the Alzheimer's Disease and Related Disorders Association) criteria and is classified as definitive (clinical diagnosis with histological confirmation), probable (typical clinical features without histological confirmation) or possible (atypical clinical features, no apparent alternative diagnosis and no histological confirmation). Diagnosis of probable AD can only be made when objective evidence of significant memory deterioration exists by neuropsychological evaluation together with at least one other biological feature such as 
abnormal CSF biomarkers and specific positron emission tomography (PET) patterns. Patients are evaluated using structured/semi-structured interviews and neuropsychological batteries with a variety of available tests to explore different aspects of cognition and behaviour. It may be advisable to apply a quick neuropsychological test to patients with apparent cognitive decline at primary care level; this would facilitate a proper canalization and management of the patient. Initial evaluations of general cognitive status can be performed using the popular Mini-Mental State Examination (MMSE) which is quick and easy to apply and provides information on global cognitive efficiency and dementia severity, although is not recommended for a definitive diagnosis.

The issue of neuropsychiatric syndromes should also be addressed during dementia evaluation as their presence is associated with a rapid deterioration of cognition. It is important to notice that $\mathrm{AD}$ and cerebrovascular disease frequently coexist and the latter strongly determines presence and severity of clinical symptoms; thus, taking into account vascular risk factors and focal neurological signs as well as Hachinski Ischemic Scale results, combined with neuroimaging, serve as powerful tools to uncover a mixed dementia, especially in elderly individuals.

The most prominent feature of $\mathrm{AD}$ is a decline in cognitive function initially characterized by deficient memory for recent events, unusually repetitive omissions and difficulty to learn new information. The Free and Cued Selective Reminding Test (FCSRT) is a useful tool to explore these initial deficiencies in suspected AD patients. Temporo-spatial disorientation appears in early stages of the disease and progresses towards intermediate stages, where the patient can be disoriented in familiar places and aphasia appears with a decrease in verbal comprehension and nominal difficulty. To evaluate the fluency and coherence of language simple and complex orders and naming tests are used.

Confident neuropsychological markers of AD in early stages are deficiencies in episodic memory. Neuropsychological evaluation of AD patients also finds a loss of autonomy with disease progression from higher level to basic daily activities, for which daily living activities tests are applied. Instrumental functions such as language, praxis and visuospatial skills start being affected in intermediate stages of AD. Visuospatial dysfunction is a common feature in this stage of disease and can be evaluated by drawing and copying tests. In moderate to severe stages, difficulty to use objects and dressing apraxia can be observed as well as visual agnosia and a visual processing dysfunction recognized by facial and object recognition tests. Working memory and attention are usually affected by the time of diagnosis.

Deficiency in activities of daily living due to cognitive decline is essential diagnostic criteria for dementia and has great impact on quality of life. Neuropsychological tests to evaluate this feature allow differentiation of dementia from mild cognitive impairment (MCI). These tests measure basic (e.g. dressing, hygiene, feeding) and instrumental (e.g. cooking, cleaning, money management) activities. Activities of the Daily Living (ADL) and Instrumental Activities of the Daily Living (IADL) are widely used scales and provide the advantage of being easily applicable in primary care. 
Non-cognitive, psychiatric and behavioural alterations common to dementia such as apathy, aggression, depression, psychosis and sleep disorders vary according to disease severity and may fluctuate; they can also present as initial manifestations of dementia. It is believed that neuropsychiatric alterations may even serve as clinical indicators of MCI conversion to AD. Scales for mood and behaviour not only evaluate the presence/absence of symptoms but their frequency, severity and impact; they can be applied to family and caregivers. The Neuropsychiatric Inventory can evaluate up to 10 behavioural alterations and is considered a standard tool, although a number of different batteries are available for this purpose. In Table 2 we provide a list of neuropsychological tests and batteries available to evaluate different aspects of cognitive and non-cognitive alterations.

\begin{tabular}{|c|c|}
\hline \multirow[t]{15}{*}{ General cognitive function } & Cognitive Assessment System (CAS) \\
\hline & Dementia Rating Scale-2 (DRS-2) \\
\hline & Kaplan Baycrest Neurocognitive Assessment (KBNA) \\
\hline & Kaufman Brief Intelligence Test (K-BIT) \\
\hline & Mini-Mental State Examination (MMSE) \\
\hline & National Adult Reading Test (NART) \\
\hline & Neuropsychological Assessment Battery (NAB) \\
\hline & Raven's Progressive Matrices (RPM) \\
\hline & Repeatable Battery for the Assessment of Neuropsychological Status (RBANS) \\
\hline & Stanford-Binet Intelligence Scales - 5th edition (SB5) \\
\hline & The Test of Nonverbal Intelligence-3 (TONI-3) \\
\hline & The Speed and Capacity of Language Processing Test (SCOLP) \\
\hline & Wechsler Abbreviated Scale of Intelligence (WASI) \\
\hline & Wechsler Adult Intelligence Scale-III (WAIS-III) \\
\hline & Woodcock-Johnson III Tests of Cognitive Abilities (WJ III COG) \\
\hline \multirow[t]{13}{*}{ Executive function } & Behavioural Assessment of the Dysexecutive Syndrome (BADS) \\
\hline & CANTAB \\
\hline & Category Test (CT) \\
\hline & Cognitive Estimation Test (CET) \\
\hline & Delis-Kaplan Executive Function System (D-KEFS) \\
\hline & Design Fluency Test \\
\hline & Five Point Test \\
\hline & Hayling and Brixton Tests \\
\hline & Ruff Figural Fluency Test (RFFT) \\
\hline & Self-Ordered Pointing Test (SOPT) \\
\hline & Stroop Test \\
\hline & Verbal Fluency \\
\hline & Wisconsin Card Sorting Test (WCST) \\
\hline \multirow[t]{4}{*}{ Attention } & Brief Test of Attention (BTA) \\
\hline & Colour Trails Test (CTT) \\
\hline & Comprehensive Trail Making Test (CTMT) \\
\hline & Conners' Continuous Performance Test II (CPT-II) \\
\hline
\end{tabular}




\begin{tabular}{|c|c|}
\hline & Integrated Visual and Auditory Continuous Performance Test (IVA + Plus) \\
\hline & Paced Auditory Serial Addition Test (PASAT) \\
\hline & Ruff 2 \& 7 Selective Attention Test (2 \& 7 Test) \\
\hline & Symbol Digit Modalities Test (SDMT), \\
\hline & Test of Everyday Attention (TEA) \\
\hline & Test of Variables of Attention (T.O.V.A.) \\
\hline & Trail Making Test (TMT) \\
\hline \multirow[t]{16}{*}{ Memory } & Autobiographical Memory Interview (AMI) \\
\hline & Benton Visual Retention Test (BVRT-5) \\
\hline & Brief Visuospatial Memory Test-Revised (BVMT-R) \\
\hline & Brown-Peterson Task \\
\hline & Buschke Selective Reminding Test (SRT) \\
\hline & California Verbal Learning Test-II (CVLT-II) \\
\hline & Doors and People Test (DPT) \\
\hline & Hopkins Verbal Learning Test-Revised (HVLT-R) \\
\hline & Recognition Memory Test (RMT) \\
\hline & Rey-Osterrieth Auditory Verbal Learning Test (RAVLT) \\
\hline & Rey Complex Figure Test (ROCF) \\
\hline & Rivermead Behavioural Memory Test-2th edition (RBMT-II) \\
\hline & Ruff-Light Trail Learning Test (RULIT) \\
\hline & Sentence Repetition Test \\
\hline & Wechsler Memory Scale-3th edition (WMS-III) \\
\hline & Wide Range Assessment of Memory and Learning - 2th edition (WRAML2) \\
\hline \multirow[t]{8}{*}{ Language } & Boston Diagnostic Aphasia Examination - 3th edition (BDAE-3) \\
\hline & Boston Naming Test-2 (BNT-2) \\
\hline & Dichotic listening - Words \\
\hline & Expressive One-Word Picture Vocabulary Test-3th edition (EOWPVT3) \\
\hline & Expressive Vocabulary Test (EVT) \\
\hline & Multilingual Aphasia Examination (MAE) \\
\hline & Peabody Picture Vocabulary Test-3th edition (PPVT-III) \\
\hline & Token Test (TT) \\
\hline \multirow[t]{7}{*}{ Visual perception } & Balloons Test \\
\hline & Bells Cancellation Test \\
\hline & Clock Drawing Test (CDT) \\
\hline & Facial Recognition Test (FRT) \\
\hline & Hooper Visual Organization Test (VOT) \\
\hline & Judgement of Line Orientation (JLO) \\
\hline & Visual Object and Space Perception Battery (VOSP) \\
\hline \multirow{5}{*}{$\begin{array}{l}\text { Somatosensory and } \\
\text { olfactory function, body } \\
\text { orientation }\end{array}$} & Finger localization \\
\hline & Right-Left Orientation (RLO) \\
\hline & Rivermead Assessment of Somatosensory Performance (RASP) \\
\hline & Smell Identification Test (SIT) \\
\hline & Tactual Performance Test (TPT) \\
\hline
\end{tabular}




\begin{tabular}{ll}
\hline Motor function & Finger Tapping Test (FTT) \\
& Grip Strength \\
& Grooved Pegboard \\
& Purdue Pegboard Test \\
\hline Mood, personality and & Beck Depression Inventory - 2th edition (BDI-II) \\
adaptive functions & Behaviour Rating Inventory of Executive Function (BRIEF) \\
& Geriatric Depression Scale (GDS) \\
& Instrumental Activities of Daily Living (IADL) \\
& Minnesota Multiphasic Personality Inventory-2 (MMPI-2) \\
& Personality Assessment Inventory (PAI) \\
& Scales of Independent Behaviour-Revised (SIB-R) \\
& Trauma Symptom Inventory (TSI) \\
Activities of the Daily Living (ADL) & Instrumental Activities of the Daily Living (IADL) \\
living & Disability Assessment for Dementia Scale \\
& Alzheimer Disease Cooperative Study ADL Scale \\
Functional Activities Questionnaire & Progressive Deterioration Scale \\
\hline
\end{tabular}

Source: Strauss E, Sherman EMS, Spreen O. A compendium of neuropsychological tests: administration, norms, and commentary, 3rd ed. New York: Oxford University Press; 2006.

Table 2. Tests and batteries for neuropsychological evaluation.

\subsection{Neuroimaging}

Imaging of the brain can reflect anatomical and physiological changes related to specific pathological processes. Structural neuroimaging technologies such as computed tomography (CT) and magnetic resonance imaging (MRI) provide relevant information on brain structures and help to exclude treatable conditions while functional neuroimaging including single photon emission computed tomography (SPECT), PET and functional magnetic resonance imaging (fMRI) informs about brain activity status. CT and MRI on AD evaluation are used to exclude neurosurgical lesions (tumors, subdural hematomas), search for evidence of cerebrovascular lesions (stroke, white matter lesions) and identify medial temporal lobe atrophy. The overlap of whole-brain atrophy in AD with normal aging and other dementias is considerable and therefore lacks diagnostic value in clinical practice. Absolute values of glucose metabolism in the hippocampus are normal in early stages of $\mathrm{AD}$ but decrease progressively during disease course as detected by PET. Hypometabolism in the associative parietal cortex, external temporal area, precuneus, posterior cingulate cortex and dorsolateral frontal cortex can be found in patients with clear dementia symptoms.

$\mathrm{AD}$ is characterized by changes in neurotransmission correlating with cognitive decline, particularly acetylcholine. A few PET tracers to measure acetylcholinesterase (AChE) and ligands to muscarinic and nicotinic receptors have been developed on the basis of the role of the cholinergic system in cognition and AD. AChE activity can be measured by its radioactively 
tagged analogues $\mathrm{N}-\left[{ }^{11} \mathrm{C}\right]$-methyl-piperidine-4-yl-propionate $\left({ }^{11} \mathrm{C}-\mathrm{PMP}\right)$ and $\mathrm{N}-\left[{ }^{11} \mathrm{C}\right]$-methylpiperidine-4-yl-acetate $\left({ }^{11} \mathrm{C}-\mathrm{MP} 4 \mathrm{~A}\right)$; nicotinic receptors can be measured by ${ }^{11} \mathrm{C}$-nicotine. A decrease in ${ }^{11} \mathrm{C}$-nicotine correlates with cognition measured by MMSE [12]. Visualization of amyloid plaques started some 15 years ago initially using $A \beta$ monoclonal antibodies and peptidic fragments and later small radioactively tagged Congo red, chrysamine $G$ and thioflavin analogues for PET and SPECT. ${ }^{18} \mathrm{~F}$ tagging may present some advantages for clinical applications compared to ${ }^{11} \mathrm{C}$. Studies have shown there is less correlation between cognitive decline and the amount of amyloid plaques in the AD brain than between cognitive decline and the amount of neurofibrillary tangles and/or neurotransmitter activity as amyloid deposits are observed in up to $30 \%$ of cognitively healthy aged individuals [13]. An increase in microglial activation has also been reported in AD patients using neuroimaging technologies [14].

\subsection{Differential diagnosis}

Even when $\mathrm{AD}$ is the most common dementing syndrome in the elderly, one shall not forget that AD can share symptoms with other disorders. Medical, neurological and psychiatric conditions to which memory loss, depression, disorientation and other symptoms can be attributed must be also investigated for a differential diagnosis of dementia. When performing a diagnosis, it is important to be aware of features found during the patient evaluation providing doubt for the AD diagnosis and bear in mind that the more accurate the diagnosis is, the better the disease can be managed. AD diagnosis can be questioned when the conditions presented in Table 3 exist.

\begin{tabular}{ll}
\hline Feature & Possible cause \\
\hline $\begin{array}{l}\text { Early alterations of behaviour: inappropriate social behaviour/feeding } \\
\text { alterations }\end{array}$ & Fronto-temporal dementia (FTD) \\
\hline Early extrapyramidal signs & Dementia with Lewy Bodies \\
Early visual hallucinations & (DLB) \\
Early visuospatial and attention deterioration & \\
Behavioural disturbances during REM sleep & \\
Fluctuation of symptoms & \\
\hline $\begin{array}{l}\text { Vascular lesions in neuroimaging } \\
\text { Sudden onset of symptoms }\end{array}$ & \\
Focal neurological signs & \\
\hline Early language alterations: & Non-fluent progressive aphasia \\
-Hesitant, forced speech, agrammatism & Semantic dementia \\
-Progressive loss of knowledge about words and objects-Progressive & Logopenic progressive aphasia \\
decline of speech with anomia & \\
\hline $\begin{array}{l}\text { Onset with high order visuospatial dysfunction/Balint syndrome: ocular } \\
\text { apraxia, optic ataxia, simultagnosia }\end{array}$ & Progressive posterior cortical atrophy \\
\hline
\end{tabular}

Table 3. Evaluation findings suggesting a diagnosis different from AD. 


\section{The search for AD biomarkers}

Biomarkers for LOAD could help predict and diagnose the disease as well as follow its progression, evaluate treatments and find new therapeutic targets. Neuroimaging technologies, genomics, transcriptomics and proteomics approaches are being extensively used globally to search for novel biomarkers for AD capable of detecting changes in the brain and peripheral tissues occurring early in the disease. Nevertheless, the finding of biomarkers with good sensitivity and specificity for $\mathrm{AD}$, and furthermore, their validation, is challenging, reason why different combinations of biomarkers, cognitive markers and risk factors may represent a more suitable tool to pursue diagnostic sensitivity and specificity for LOAD.

\subsection{Brain biomarkers}

Markers of AD pathology have been extensively investigated in the brain of these patients; unfortunately, the task has been challenging due to overlap of a number of these findings with other brain disorders and even with normal ageing, as is the case of whole-brain atrophy. Although some findings might still represent specific biomarkers for AD as shown by a recent study where a decrease in grey matter diffusion values observed mainly in the posterior cingulate gyrus and precuneus area has been suggested as a potential new biomarker for AD in early stages [15].

However, abnormalities in brain imaging (structural and functional), cognitive markers (neuropsychological scores) and molecules (mRNA, proteins) measured in specific brain regions affected by $\mathrm{AD}$ are currently demonstrating a better potential to identify $\mathrm{AD}$ and differentiate it from other disorders in a number of different combinations. Recent studies on the Alzheimer's Disease Neuroimaging Initiative (ADNI) subjects have shown correlations between temporal lobe atrophy measured by serial MRI scans with CSF p-tau and tau/A $\beta_{42}$ ratio as well as with cognitive markers which apparently may also be able to predict MCI-AD conversion. Hippocampal volume loss showed to correlate with ApoE4 genotype. PET imaging using ${ }^{18} \mathrm{~F}$-fludeoxyglucose (FDG) showed correlations with $\mathrm{A} \beta_{42}$, but using ${ }^{11} \mathrm{C}$ labelled Pittsburgh compound B $\left({ }^{11} \mathrm{C}-\mathrm{PIB}\right)$ to specifically bind fibrillar $\mathrm{A} \beta$ plaques showed correlations not only with $\mathrm{A} \beta_{42}$ but $\mathrm{t}$-tau and $\mathrm{p}$-tau $\mathrm{t}_{181}$ [16]. However, recently, a comparative study suggested ADNI subjects appear to have a more aggressive pathology than populationbased samples, as observed by the rates of decline in hippocampal volume measured by MRI, which may raise concerns about ADNI subjects not being representative of the general population [17]. In any case, we must remember all findings should be validated in different cohorts and here may be the point where reproducibility cannot be achieved, reason why this has become a very active field in AD research.

\subsection{Peripheral biomarkers}

The search for easily available biomarkers for AD has lead scientists in the past years to investigate a number of molecules in CSF and blood components. From a variety of these studies several molecules were proposed as potential blood biomarkers for the disease (Table 4), although some results remained controversial. Until now, the best biomarker validated for 
$\mathrm{AD}$ diagnosis is the $\mathrm{CSF}$ tau/A $\beta_{42}$ ratio. Other biomarkers in CSF have been investigated though and, recently, protein markers of DNA damage have shown potential as biomarkers for AD and other dementias [18].

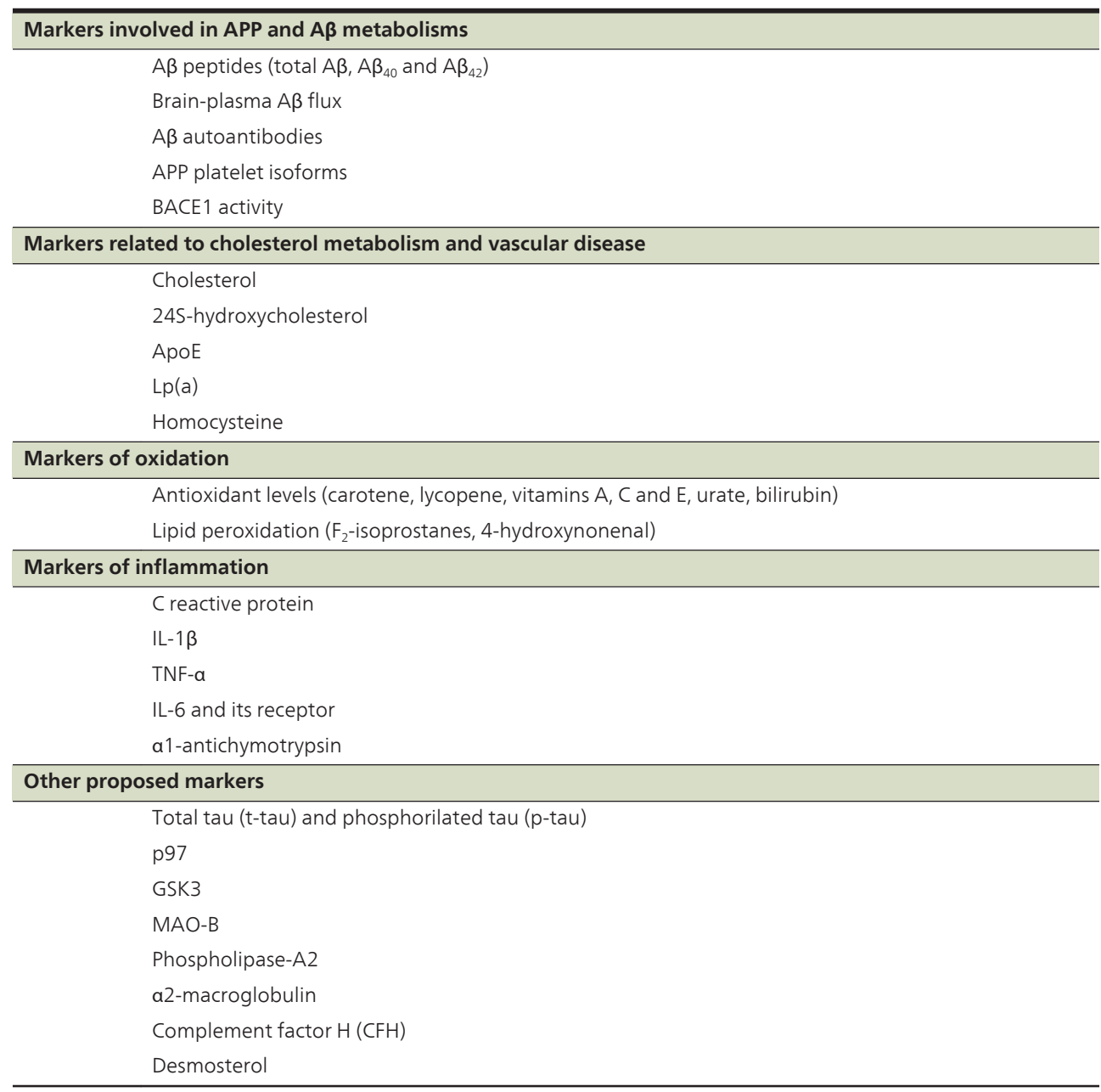

Table 4. Proposed blood biomarkers for AD.

In recent years, the approach for biomarker discovery has changed, now focusing on pattern recognition and combinations of different markers to identify panels capable of differentiating $\mathrm{AD}$ from healthy control subjects and, in some cases, from other brain disorders. As the work on this field has been so extensive we will limit ourselves now to briefly mention some of the recent biomarker findings reported principally in blood plasma (Table 5), where protein profiles have been extensively analysed in $\mathrm{AD}, \mathrm{MCI}$ and control subjects using a wide range 
of technologies. We shall not forget serum and blood cells are also a potential source of pathologic alterations reflecting disease.

\begin{tabular}{|c|c|c|c|c|}
\hline Sample & Cohort & Panel & Change & Ref. \\
\hline \multirow[t]{2}{*}{ Plasma } & $\begin{array}{l}\mathrm{AD} \text { and controls } \\
\text { from the Australian } \\
\text { Imaging Biomarker } \\
\text { and Lifestyle study } \\
\text { (AIBL) }\end{array}$ & $\begin{array}{l}\text { cortisol, pancreatic polypeptide, insulin like } \\
\text { growth factor binding protein } 2, \beta 2 \\
\text { microglobulin, vascular cell adhesion molecule } \\
\text { 1, carcinoembryonic antigen, matrix } \\
\text { metalloprotein } 2, C D 40, \text { macrophage } \\
\text { inflammatory protein 1a, superoxide } \\
\text { dismutase, and homocysteine }\end{array}$ & Increase & [19] \\
\hline & & $\begin{array}{l}\text { apolipoprotein E, epidermal growth factor } \\
\text { receptor, hemoglobin, calcium, zinc, } \\
\text { interleukin } 17 \text {, and albumin }\end{array}$ & Decrease & \\
\hline \multirow[t]{2}{*}{ Plasma } & $\begin{array}{l}\mathrm{MCl}, \mathrm{AD} \text { and } \\
\text { controls from } \mathrm{ADNI}\end{array}$ & $\begin{array}{l}\text { eotaxin } 3 \text {, pancreatic polypeptide, } \mathrm{N} \text {-terminal } \\
\text { protein } \mathrm{B} \text {-type brain natriuretic peptide, } \\
\text { tenascin C }\end{array}$ & Increase & [20] \\
\hline & & $\operatorname{IgM}$ and $\mathrm{ApoE}$ & Decrease & \\
\hline $\begin{array}{l}\text { Peripheral blood } \\
\text { mononuclear } \\
\text { cells (PBMCs) }\end{array}$ & LOAD and controls & $\begin{array}{l}\text { fatty acid amide hydrolase (FAAH) gene } \\
\text { expression, protein levels and activity }\end{array}$ & Increase & {$[21]$} \\
\hline Plasma & $\begin{array}{l}\mathrm{MCl}-\mathrm{AD} \text { converters } \\
\text { and controls from } \\
\mathrm{ADNI}\end{array}$ & $\begin{array}{l}\text { a2-macroglobulin, angiotensinogen, } \\
\text { apolipoprotein A-II (ApoA-II), ApoE, } \\
\text { betacellulin (BTC), Fas ligand (FasL), heparin- } \\
\text { binding EGF-like growth factor (HB-EGF), } \\
\text { macrophage inflammatory protein-1a } \\
\text { (MIP-1a), peptide YY (PYY), m glutamic } \\
\text { oxaloacetic transaminase (SGOT), transthyretin } \\
\text { (TTR) }\end{array}$ & Signature & {$[22]$} \\
\hline Plasma & $\mathrm{AD}$ and controls & $\begin{array}{l}\text { ANG-2, CCL5, CCL7, CCL15, CCL18, CXCL8, } \\
\text { EGF, G-CSF, GDNF, ICAM-1, IGFBP-6, IL-1a, IL-3, } \\
\text { IL-11, M-CSF, PDGF-BB, TNF-a, TRAIL-R4 }\end{array}$ & Signature & {$[23]$} \\
\hline Plasma & $\begin{array}{l}\text { AD and controls } \\
A D \text { and other } \\
\text { dementias }\end{array}$ & $\begin{array}{l}\text { EGF, PDG-BB and MIP-1 } \delta \\
\text { TRAIL-R4 }\end{array}$ & Not stated & [24] \\
\hline Serum & $\mathrm{AD}$ and controls & miR-137, $-181 c,-9,-29 a / b$ & $\begin{array}{l}\text { Down- } \\
\text { regulated }\end{array}$ & {$[25]$} \\
\hline Plasma & $\begin{array}{l}\mathrm{MCl}, \mathrm{AD} \text { and } \\
\text { control from } \\
\text { AddNeuroMed }\end{array}$ & $\begin{array}{l}\text { complement components C3 and C3a, } \\
\text { complement factor-I, } y \text {-fibrinogen and } \\
\text { alpha-1-microglobulin }\end{array}$ & $\begin{array}{l}\text { Associate with } \\
\text { whole-brain } \\
\text { volume }\end{array}$ & [26] \\
\hline
\end{tabular}

Table 5. Recent findings on blood biomarkers for AD. 


\subsubsection{Expression of MAPT, APP, NCSTN and BACE1 in lymphocytes}

Neurofibrillary tangles and amyloid plaques are hallmarks in the AD brain involving the microtubule-associated protein tau (MAPT) and $\mathrm{A} \beta$ peptides generated from the amyloid precursor protein (APP) by sequential cleavage of the $\beta$ - and $\gamma$-secretases, nicastrin (NCSTN) being a major component of the latter. These molecules have been widely investigated in the brain; nevertheless, little is known about their expression in peripheral cells. The established role of inflammation in the AD pathology lead us to question how these major genes express in peripheral lymphocytes and, furthermore, whether their expression may correlate with common medical conditions in the elderly that have been identified as risk factors for LOAD.

\section{a. Samples}

To address this question, we collected blood samples from a total of 72 subjects, including 48 healthy individuals divided into 4 groups by age ( 25 to 92 years of age), 12 clinically diagnosed $\mathrm{AD}$ patients and 12 patients suffering from other brain disorders (vascular dementia-VaD-, Parkinson's disease-PD-, traumatic brain injury-TBI-, cerebrovascular events-CVE-, psychotic disorder) as a comparative group named NP (Table 6). All patients had been previously diagnosed by appropriate professionals in the private practice and healthy subjects volunteered, making this a small population-based cohort with exploratory purposes. For all subjects, blood pressure was measured and history was briefly collected; for all patients, interviews with family members were also performed. All patients are participants in our neuro-rehabilitation program [27] and samples were collected at the time of enrolment.

\begin{tabular}{lccl}
\hline Group & $\boldsymbol{n}$ & Mean age & Diagnosis \\
\hline I & 12 & 30 & \\
II & 12 & 44 & Cognitively healthy \\
III & 12 & 58 & \\
IV & 12 & 80 & \\
AD & 12 & 80 & LOAD \\
NP & 12 & 78 & PD, MCI, VaD, CVE, TBI, psychotic disorder/ schizophrenia \\
\hline
\end{tabular}

Table 6. Basic characteristics of the study groups.

\section{b. Methodology}

After collection, serum was separated from whole blood to quantify total cholesterol, glucose and triglycerides. Lymphocytes were isolated from EDTA whole blood using Lymphoprep (Nycomed Pharma). After washing the pellet, total RNA was extracted from lymphocytes by the TRIzol method (Invitrogen). Endpoint RT-PCR was selected to semiquantify MAPT, APP, NCSTN and the $\beta$-secretase BACE1 expression levels because of our strong interest in the use of the most easily available technologies for small research and clinical laboratories. Glyceraldehyde-3-phosphate-dehydrogenase (GAPDH) was the housekeeping gene of choice. 


\section{c. Data analysis}

Gene expression values were compared between study groups using Student's $t$ and MannWhitney's $U$ tests. Associations between gene expression levels and between expression with raw values of the analysed risk factor conditions were investigated using Spearman's correlation coefficients. Differences were considered significant at $\mathrm{p}<0.05$.

d. Main findings

As the full report of this exploratory study is currently under review for publication, we will limit ourselves here to share only the most relevant of our results. From the four genes analysed, we were not able to detect NCSTN in any AD subjects, therefore showing significant differences between the AD group and all the others, but particularly differing from the group of other brain disorders which showed the highest expression levels for this study (Figure 1). We also investigated the prevalence of commonly elevated variables increasing the risk for AD: blood pressure (taken as systolic/diastolic pressure and pulse), glucose, total cholesterol and triglycerides. High serum triglycerides and cholesterol prevailed in the whole study population whereas the AD group showed no greater prevalence of any of these conditions. Correlation analyses associated NCSTN expression in lymphocytes with that observed for APP and BACE1, as well as with serum total cholesterol levels in the whole study population but, when separated by groups, NCSTN expression only correlated with cholesterol levels in the group NP.

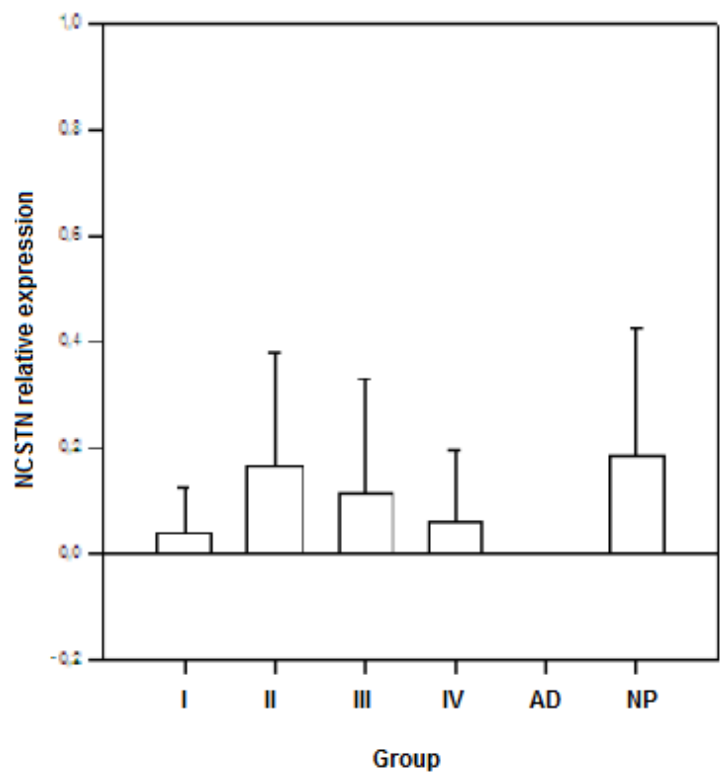

Figure 1. NCSTN relative expression in lymphocytes. 


\section{Conclusions}

Late-onset Alzheimer's disease (LOAD) is the most common dementia in the aged population and therefore great efforts to discover the factors conferring an increased risk for this disease and biomarkers able to help in its diagnosis and prognosis are made worldwide. These intensively active fields of research have produced huge amounts of information, some of which have been controversial but also a good volume showing real potential and waiting to seek further replication and validation. However, while research on biomarkers for AD struggles with the challenge of sensibility, specificity and reproducibility in different cohorts, it is of major importance to assure the best possible performance when clinically diagnosing a patient with LOAD. Clinical diagnosis of dementias may be a bit of a long process which requires some expertise and must include specific laboratory and neuropsychological tests as well as imaging of the brain and family interviews. An accurate diagnosis has implications on therapeutic approaches, disease progression and costs, quality of life of patients, family and caregivers and other important aspects for disease management.

We previously reported the establishment of a neuro-rehabilitation program for AD and other neuropsychological disorders in our locality [27] and made brief mention of a biomarker study for which now we reported results on this occasion. Our major interest resides in using the most easily and widely available technologies for biomarker discovery and therapeutic approaches which could be directed to the general population around the globe in a cost effective manner. Even when our work is in the very first stages of this research, we seek to continue our studies in larger cohorts, including more variables and using better technologies.

\section{Acknowledgements}

The author would like to thank to Gonzalo E. Aranda-Abreu, $\mathrm{PhD}$, leading researcher of the original work commented herein, and María E. Hernández Aguilar, PhD, both from the Center of Cerebral Investigations, Universidad Veracruzana. Also thanks to Consejo Nacional de Ciencia y Tecnología (CONACyT, Mexico) for the doctoral scholarship number 223277 in biomedical sciences granted to M.H.R., and the nursing home Emperatríz de las Américas.

\section{Author details}

\section{Marisol Herrera-Rivero}

Doctorate in Biomedical Sciences, Centre of Biomedical Investigations, Universidad Veracruzana, Xalapa, Veracruz, Mexico 


\section{References}

[1] Shah NS, Vidal JS, Masaki K, Petrovitch H, Ross GW, Tilley C et al. Midlife blood pressure, plasma beta-amyloid, and the risk for Alzheimer disease. Hypertension 2012; 59: 780-86. doi: 10.1161/hypertensionaha.111.178962

[2] Yang YH, Roe CM, Morris JC. Relationship between late-life hypertension, blood pressure, and Alzheimer's disease. An J Alzheimers Dis Other Demen 2011; 26(6): 457-62. doi: 10.1177/1533317511421779

[3] Rodríguez-Valdés R, Álvarez-Amador A, Aguilar-Fabré L. Vascular factors and Alzheimer's disease (in Spanish). Rev Mex Neuroci 2006; 7(3): 225-230. http://www.medigraphic.com/pdfs/revmexneu/rmn-2006/rmn063h.pdf

[4] Kalaria RN. Cerebral vessels in aging and Alzheimer's disease. Pharmacol Ther 1996; 72(3): 193-214. tp://dx.doi.org/10.1016/S0163-7258(96)00116-7

[5] Jellinger KA, Paulus W, Wrocklage C, Litvan I. Traumatic brain injury as a risk factor for Alzheimer's disease: comparison of two retrospective autopsy cohorts with evaluation of ApoE genotype. MNC Neurol 2001; 1:3. doi: 10.1186/1471-2377-1-3

[6] Uryu K, Laurer H, McIntosh T, Praticò D, Martínez D, Leight S et al. Repetitive mild brain trauma accelerates Abeta deposition, lipid peroxidation, and cognitive impairment in a transgenic mouse model of Alzheimer amyloidosis. J Neurosci 2002; 22(2): 446-54. http://www.jneurosci.org/content/22/2/446.long

[7] Sáez-Fonseca JA, Lee L, Walker Z. Long-term outcome of depressive pseudodementia in the elderly. J Affect Disord 2007; 101(1-3): 123-9. doi: 10.1016/j.jad.2006.11.004

[8] Barnes DE, Yaffe K, Byers AL, McCormick M, Schaefer C, Whitmer RA. Midlife vs late-life depressive symptoms and risk of dementia: differential effects for Alzheimer disease and vascular dementia. Arch Gen Psychiatry 2012; 69(5): 493-8. doi: 10.1001/ archgenpsychiatry.2011.1481

[9] Kang JE, Lim MM, Bateman RJ, Lee JJ, Smyth LP, Cirrito JR et al. Amyloid-beta dynamics are regulated by orexin and the sleep-wake cycle. Science 2009; 326(5955): 1005-7. doi: 10.1126/science.1180962

[10] Liberati G, Raffone A, Olivetti Belardinelli M. Cognitive reserve and its implications for rehabilitation and Alzheimer's disease. Cogn Process 2012; 13(1): 1-12. doi: 10.1007/ s10339-011-0410-3

[11] Lanyau Domínguez Y. The diet in Alzheimer's disease (in Spanish). Revista Cubana de Salud Pública 2009; 35(4): 55-64. http://redalyc.uaemex.mx/redalyc/pdf/ 214/21418848007.pdf 
[12] Nordberg A, Lundqvist H, Hartvig P, Lilja A, Langstrom B. Kinetic analysis of regional (S)(-)11C-nicotine binding in normal and Alzheimer brains. In vivo assessments using positron emission tomography. Alzh Dis Assoc Disord 1995; 9(1):21-7.

[13] Villemagne VL, Pike KE, Darby D, Maruff P, Savage G, Ng S et al. Abeta deposition in older non-demented individuals with cognitive decline are indicative of preclinical Alzheimer's disease. Neuropsychologia 2008; 46(6):1688-97. http://dx.doi.org/ 10.1016/j.neuropsychologia.2008.02.008

[14] Cagnin A, Brooks DJ, Kennedy AM, Gunn RN, Myers R, Turkheimer FE et al. In vivo measurement of activated microglia in dementia. Lancet 2001; 358(9280):461-7. doi: 10.1016/S0140-6736(01)05625-2

[15] Jacobs HI, van Boxtel MP, Gronenschild EH, Uylings HB, Jolles J, Verhey FR. Alzheimers Dement 2012. doi: 10.1016/j.jalz.2011.11.004

[16] Trojanowski JQ, Trojanowski JQ, Vandeerstichele H, Korecka M, Clark CM, Aisen PS et al. Update on the biomarker core of the Alzheimer's Disease Neuroimaging Initiative subjects. Alzheimers Dement 2010; 6(3): 230-8. doi: 10.1016/j.jalz.2010.03.008

[17] Whitwell JL, Wiste HJ, Weigand SD, Rocca WA, Knopman DS, Roberts RO et al. Comparison of imaging biomarkers in the Alzheimer Disease Neuroimaging Initiative and the Mayo Clinic Study of Aging. Arch Neurol 2012; 69(5): 614-44. doi: 10.1001/archneurol.2011.3029

[18] Watabe-Rudolph M, Song Z, Lausser L, Schnack C, Begus-Nahrmann Y, Scheithauer $\mathrm{MO}$ et al. Chitinase enzyme activity in CSF is a powerful biomarker of Alzheimer disease. Neurology 2012; 78(8): 569-77. doi: 10.1212/WNL.0b013e318247caa1

[19] Doecke JD, Laws SM, Faux NG, Wilson W, Burnham SC, Lam CP et al. Blood-based protein biomarkers for diagnosis of Alzheimer Disease. Arch Neurol 2012. doi: 10.1001/archneurol.2012.1282

[20] Soares HD, Potter WZ, Pickering E, Kuhn M, Immermann FW, Shera DM et al. Plasma biomarkers associated with the Apolipoprotein E genotype and Alzheimer disease. Arch Neurol 2012. doi: 10.1001/archneurol.2012.1070

[21] D'Addario C, Di Francesco A, Arosio B, Gussago C, Dell'osso B, Bari M et al. Epigenetic regulation of fatty acid amide hydrolase in Alzheimer disease. PLoS One 2012; 7(6): e39186. doi: 10.1371/journal.pone.0039186

[22] Johnstone D, Milward EA, Berretta R, Moscato P. Multivariate protein signatures of pre-clinical Alzheimer's disease in the Alzheimer's disease neuroimaging initiative (ADNI) plasma proteome dataset. PLoS One 2012; 7(4): e34341. doi: 10.1371/journal.pone.0034341

[23] Ray S, Britschgi M, Herbert C, Takeda-Uchimura Y, Boxer A, Blennow K et al. Classification and prediction of clinical Alzheimer's diagnosis based on plasma signaling proteins. Nat Med 2007; 13(11): 1359-62. doi: 10.1038/nm1653 
[24] Björkqvist M, Ohlsson M, Minthon L, Hansson O. Evaluation of a previously suggested plasma biomarker panel to identify Alzheimer's disease. PLoS One 2012; 7(1): e29868. doi: 10.1371/journal.pone.0029868

[25] Geekiyanage H, Jicha GA, Nelson PT, Chan C. Blood serum miRNA: non-invasive biomarkers for Alzheimer's disease. Exp Neurol 2012; 235(2): 491-6. doi: 10.1016/ j.expneurol.2011.11.026

[26] Thambisetty M, Simmons A, Hye A, Campbell J, Westman E, Zhang Y et al. Plasma biomarkers of brain atrophy in Alzheimer's disease. PLoS One 2011; 6(12): e28527. doi: 10.1371/journal.pone.0028527

[27] Herrera-Rivero M, Aranda-Abreu GE. Therapeutics of Alzheimer's Disease. In: Advanced Understanding of Neurodegenerative Diseases, Raymond Chuen-Chung Chang (Ed.), 2011. ISBN: 978-953-307-529-7, InTech. Available from: http://www.intechopen.com/books/advanced-understanding-of-neurodegenerative-diseases/therapeuticsof-alzheimer-s-disease 
